\title{
Do Preferred Beat Rate and Entrainment to the Beat Have a Common Origin in Movement?
}

\author{
LAUREL J. TRAINOR \\ McMaster University [1]
}

\begin{abstract}
The idea that beat induction derives from body movement is attractive, but until recently unequivocal empirical evidence of such a linkage has been elusive. Todd et al. (2007) provide evidence that individual differences in locomotion affect preferred beat rate, and Phillips-Silver \& Trainor $(2005,2007$, under review) show that metrical interpretation is affected by movement. These studies examine different aspects of rhythm processing, but together provide converging evidence for pervasive movement/auditory interactions.
\end{abstract}

Submitted 2007 January 12; accepted 2007 January 12

KEYWORDS: rhythm, perception, beat induction, entrainment

MUSICAL beat induction-the perceptual extraction of a regular underlying beat pattern from a temporal sound pattern-is perhaps the most fundamental prerequisite for the emergence of musical behaviour. The process of beat induction has therefore been of great interest to researchers studying the evolutionary and cultural origins of music (Clark, 1999; Cross, 2003; Fitch, 2005; Molinari, Leggio, De Martin, Cerasa, \& Thaut, 2003; Todd, Cousins, \& Lee, 2007). The hypothesis that musical beat induction derives from body movement is attractive because the tempo range of optimal musical pulse perception, around 300-900 ms onset-to-onset, is similar to that of locomotion (Fraisse, 1982). Furthermore, rhythmic body movement is evolutionarily ancient, and music and dance are inseparable in many human cultures (Wallin, Merker, \& Brown, 2000).

However, it is not immediately obvious how to test the hypothesis that feeling the beat derives from body movement. Until recently, there was little empirical evidence for a direct link between musical beat induction and body movement, but two interesting approaches have been presented in the last couple of years. Todd, Cousins, \& Lee (2007) measured individual differences in anthropometric factors that affect the tempo of locomotion, and showed that these individual differences vary with individual differences in auditory perceptual preferred beat rate, suggesting that preferred beat rate is related to rhythmic body movement. Phillips-Silver and Trainor (2005, 2007, under review) showed that body movement on either every second or every third beat of an ambiguous auditory rhythm pattern (i.e., a pattern without physical accents) affects whether the beat of the auditory pattern is perceived as being in duple or triple time, thus demonstrating a multisensory connection between movement and auditory beat induction.

The methodologies of Todd et al. (2007) and Phillips-Silver \& Trainor (2005, 2007, under review) are very different, and together they provide quite convincing converging evidence for movement/auditory interactions for musical beat perception. However, because the two approaches are quite different in their emphasis, a comparison between the two can be instructive in considering the mechanisms involved in the interaction, the levels at which they operate, and how they develop. Todd et al. focus on preferred beat rates and individual differences, an emphasis that allows the examination of correlations between perceptual preferences and anthropometric factors that affect locomotion tempos. A direct measure of preferred walking speed might have revealed a stronger correlation with perception, but in any case the emphasis is on characterizing each individual with a single tempo. On the other hand, the approach of Phillips-Silver and Trainor focuses on the fact that the same individual can feel a beat at different tempos, and that different tempos of body movement can influence the rate at which the beat is perceived. 
It is possible that the data from Todd et al. (2007) reflects a relatively primitive multisensory mechanism connecting the speed of personal locomotion with auditory tempo, whereas the data from Phillips-Silver \& Trainor $(2005,2007$, under review) reflects a mechanism for beat induction that is relatively unique to humans. It has been suggested that one of the abilities underlying musical behaviour that might distinguish humans from most other species is the ability to entrain to beats with different tempos (Fitch, 2005). From infancy, people readily recognize a melody or rhythm pattern when it is produced at different speeds (Trehub \& Thorpe, 1989) just as they recognize a melody when it is transposed to different pitch levels (e.g., Trainor, 2005; Trainor \& Trehub, 1992; Plantinga \& Trainor, 2005). In fact, recognition of rhythmic invariance across tempo change is critical for musical behaviour. Yet there is no good direct evidence that other species are able to do this. Some apes (e.g., chimpanzees) and birds (e.g., woodpeckers) do engage in drumming, but there is no evidence that they can entrain to an external beat and vary their tempo accordingly (Fitch, 2005). In this context, the preferred beat rates of individual people as measured by Todd et al., and their relation to anthropometric features, may well reflect a mechanism shared with many other species who produce rhythmic behaviours. On the other hand, the auditory-movement interactions demonstrated in Phillips-Silver and Trainor's research rely on the ability to perceive and entrain to different tempos: the ability to hear either a faster beat by perceiving every second beat of a physically unaccented pattern as accented, or to hear a slower beat by perceiving every third beat as accented, presupposes the ability to perceive different tempos and to entrain to different tempos. It also presupposes the ability to perceptually group sound events into a rhythmic hierarchy; either two or three beats at one tempo level are grouped together to form a second slower tempo level at either one half or one third of the primary tempo, respectively. Thus, there may be a species-general mechanism for preferred auditory pulse rate that is connected to locomotion, and a human-specific, or at least more rare, beat entrainment mechanism that can operate at hierarchical levels, but is also influenced by movement.

For both the proposed species-general and species-specific mechanisms of movement-auditory interactions, it is crucial to understand what aspects of movement are involved. Movement systems are complex and include motor planning, tactile, proprioception, and vestibular inputs. Recent work suggests that the vestibular system may be particularly important. Phillips-Silver \& Trainor $(2005 ; 2007)$ found that active movement while listening to the ambiguous rhythm pattern (whether blind-folded or sighted) was necessary in both infants and adults for biasing whether they interpreted the ambiguous auditory rhythm in duple or triple meter. Simply watching another person move to the ambiguous rhythm did not bias the auditory interpretation. Furthermore, passive movement of adults' legs on every second or on every third beat, which does not stimulate the vestibular system, did not affect auditory interpretation of the ambiguous rhythm. On the other hand, movement of the head alone, which does stimulate the vestibular system, did bias the auditory interpretation of the metrical pattern (Phillips-Silver \& Trainor, under review; PhillipsSilver \& Trainor, 2006). Thus, input from the vestibular system appears to be crucial for the process of beat entrainment that is potentially unique to humans. The role of the vestibular system in the influence of locomotion on preferred auditory tempo is not yet known, but given that the vestibular system is evolutionarily ancient, it would be a good candidate mechanism to consider for transfer of locomotion tempo to auditory beat preference.

It is also of interest to consider the ontogenetic origins of auditory-movement interactions. Are these interactions innate in that they are genetically specified, or does concurrent correlated auditory and movement input experience cause the two systems to become wired together? While young infants are not able to self-locomote, they do experience considerable daily correlated multisensory input. For example, caregivers rock them while singing; and while being held, infants experience the movement of the caregiver's walking concurrently with the sound of her footsteps. Furthermore, the vestibular system develops early (Clark, Kreutzberg, \& Chee, 1977), as evidenced by infants' delight in being bounced, swooped, and rocked. The fact that infants show multisensory connections between movement and auditory rhythm suggests that self-locomotion is not necessary for the effect of movement on metrical interpretation and auditory entrainment, but that experience with correlated vestibular and auditory input may well establish the interaction between movement and auditory metrical interpretation.

It has been proposed that there is an intrinsic central resonance frequency of motion set to around $2 \mathrm{~Hz}$ for all people, regardless of anthropomorphic characteristics and biomechanical resonances, that can be seen in head, hip, wrist and ankle movements recorded in naturalistic settings throughout the day (MacDougall \& Moore, 2005). However, the variation seen between people in Todd et al.'s (2007) data suggests that it is likely that the relation between individual locomotion tempo and preferred auditory tempo is established at least in part through experience. Indeed, it would be hard to imagine a genetically- 
based mechanism that would uniquely match anthropomorphic features to auditory preferences. That said, there are interesting developmental questions concerning the plasticity of the process. Presumably, as the child grows, preferred tempo should slow down, and some data suggests that this is the case (MacAuley, Jones, Holub, Johnston, \& Miller, 2006). It would be interesting to conduct a stricter test of this hypothesis in a longitudinal study in which the correlation between changes over time in anthropometric features and preferred auditory tempo was determined. The infancy stage is also of interest in this regard, because although young infants do not self-locomote, they spend much time locomoting with their caregivers. If the vestibular system is key to the interaction, the prediction would be made that young infants should prefer the tempo corresponding to the anthropomorphic features of their primary caregivers. Finally, it would be interesting to consider the dynamics of the plasticity of the interaction. Todd et al. (2007) found more variability and more slowing of preferred tempo with age in males than in females. Because most infants are carried primarily by women, the early experience of female infants is closer to their future adult experience with self-locomotion than is the early experience of male infants. The long-term effect, if any, of this initial experience is unknown.

In summary, the research programs of Todd et al. (2007) and Phillips-Silver and Trainor (2005, 2007, under review) provide converging evidence for strong multi-sensory interactions between movement and auditory rhythm, although the two approaches may be investigating different levels of the interaction. Interesting questions remain as to the mechanisms by which these interactions operate and develop, the extent to which they are species general, and their importance as an ability underlying musical behaviour in humans.

\section{NOTE}

[1] Address correspondence to Laurel J. Trainor / Department of Psychology, Neuroscience, \& Behaviour / McMaster University / Hamilton, ON / Canada L8S 4K1 / LJT@mcmaster.ca / 905-525-9140 ext. 23007 / fax 905-529-6225

\section{ACKNOWLEDGEMENTS}

This paper was supported by a grant from the Natural Sciences and Engineering Research Council of Canada. I thank Lisa Hotson for comments on an earlier draft.

\section{REFERENCES}

Clark, E. F. (1999). Rhythm and timing in music. In D. Deutsch (Ed.), The Psychology of Music, $2^{\text {nd }}$ edition. San Diego: Academic Press.

Clark, D. L., Kreutzberg, J. R., \& Chee, F. K. (1977). Vestibular stimulation influences motor development in infants. Science, Vol. 196, pp. 1228-1229.

Cross, I. (2003). Music, cognition, culture and evolution. In I. Peretz, R. Zatorre (Eds.), The Cognitive Neuroscience of Music (pp. 42-56). New York, NY, US: Oxford University Press.

Fitch, T. (2005). The evolution of music in comparative perspective. In G., Avanzini, L. Lopez, S. Koelsch, \& M. Manjno, (Eds.), The Neurosciences and Music II: From Perception to Performance (pp. 2949). New York: New York Academy of Sciences.

Fraisse, P. (1982). Rhythm and tempo. In D. Deutsch (Ed.), The Psychology of Music (pp. 149-180). New York: Academic Press.

MacDougall, H. G., \& Moore, S. T. (2005). Marching to the beat of the same drummer: The spontaneous tempo of human locomotion. Journal of Applied Physiology, Vol. 99, pp. 1164-1173. 
McAuley, J. D., Jones, M. R., Holub, S., Johnston, H. M., \& Miller, N. S. (2006). The time of our lives: Life span development of timing and event tracking. Journal of Experimental Psychology: Human Perception and Performance, Vol. 135, pp. 348-367.

Molinari, M., Leggio, M. G., De Martin, M., Cerasa, A., \& Thaut, M. (2003). Neurobiology of rhythmic motor entrainment. Annals of the New York Academy of Science, Vol. 999, pp. 313-321.

Phillips-Silver, J., \& Trainor, L. J. (2006, August). Adults hear the rhythm they feel through active and passive body movement. Poster session presented at the $9^{\text {th }}$ International Conference on Music Perception and Cognition, Bologna, Italy.

Phillips-Silver, J., \& Trainor, L. J. (2005). Feeling the beat: Movement influences infants' rhythm perception. Science, Vol. 308, pp. 1430.

Phillips-Silver, J., \& Trainor, L. J. (2007). Hearing what the body feels: Auditory encoding of rhythmic movement. Cognition, doi:10.1016/j.cognition.2006.11.006.

Phillips-Silver, J., \& Trainor, L. J. (under review). Multisensory rhythm perception: Vestibular influences on auditory metrical interpretation.

Plantinga, J., \& Trainor, L. J. (2005). Memory for melody: Infants use a relative pitch code. Cognition, Vol. 98, pp. 1-11.

Todd, N. P., Cousins, R., \& Lee, C. S. (2007). The contribution of anthropometric factors to individual differences in the perception of rhythm. Empirical Music Review, Vol. 2 No. 1, pp. 1-13.

Todd, N. P. McA., Cousins, R., \& Lee, C.S. (2007). The contribution of anthropometric factors to individual differences in the perception of rhythm. Empirical Musicology Review, Vol. 2 No. 1, pp. 1-13.

Trainor, L. J. (2005). Are there critical periods for musical development? Developmental Psychobiology, Vol. 46, pp. 262-278.

Trainor, L. J., \& Trehub, S. E. (1992). A comparison of infants' and adults' sensitivity to Western tonal structure. Journal of Experimental Psychology: Human Perception and Performance, Vol. 18, pp. 394402.

Trehub, S. E., \& Thorpe, L. A. (1989). Infants' perception of rhythm: Categorization of auditory sequences by temporal structure. Canadian Journal of Psychology, Vol. 43, pp. 217-229.

Wallin, N. L., Merker, B., \& Brown, S. (2000). The Origins of Music. Cambridge, MA: MIT Press. 